Article

\title{
Low-Temperature Crystal Structures of the Hard Core Square Shoulder Model
}

\author{
Alexander Gabriëlse, Hartmut Löwen and Frank Smallenburg * \\ Institut für Theoretische Physik II: Weiche Materie, Heinrich-Heine-Universität Düsseldorf, Universitätsstr. 1, \\ 40225 Düsseldorf, Germany; a.l.gabrielse@gmail.com (A.G.); hartmut.loewen@uni-duesseldorf.de (H.L.) \\ * Correspondence: f.smallenburg@gmail.com
}

Received: 30 September 2017; Accepted: 3 November 2017; Published: 7 November 2017

\begin{abstract}
In many cases, the stability of complex structures in colloidal systems is enhanced by a competition between different length scales. Inspired by recent experiments on nanoparticles coated with polymers, we use Monte Carlo simulations to explore the types of crystal structures that can form in a simple hard-core square shoulder model that explicitly incorporates two favored distances between the particles. To this end, we combine Monte Carlo-based crystal structure finding algorithms with free energies obtained using a mean-field cell theory approach, and draw phase diagrams for two different values of the square shoulder width as a function of the density and temperature. Moreover, we map out the zero-temperature phase diagram for a broad range of shoulder widths. Our results show the stability of a rich variety of crystal phases, such as body-centered orthogonal (BCO) lattices not previously considered for the square shoulder model.
\end{abstract}

Keywords: colloidal crystals; hard core square-shoulder model; cell theory; phase behavior; computer simulation

\section{Introduction}

In the past several decades, the principles of designed colloidal self-assembly [1,2] have been widely used to generate novel structures on the mesoscale by tailored interactions [3,4] and external stimuli [5-7]. The colloidal building blocks in this framework can have either spherical [8-10] or asymmetric shape (for example, see Refs. [11-13]), and can range in size from the micrometer scale down to a few nanometers, where their shape and size can be controlled with atomic precision [14]. Regardless of shape or size, these particles self-assemble due to a combination of thermal noise, mutual interactions, and external forces. In order to predict and control colloidal self-assembly, it is crucial to understand the equilibrium bulk phase diagram for a given colloidal interaction.

This framework of self-assembly provides an effective route towards the creation of an amazing range of colloidal crystal structures by tuning the interactions between colloidal building blocks. Even in the seemingly simple case of monodisperse particles with spherically symmetric interactions, an impressive array of different structures have been both predicted theoretically and observed in experiments. In particular, it has been shown that soft repulsive interaction potentials can be tuned to favor e.g., open crystal lattices such as diamond [15,16], lattices with large unit cells such as A15 [17], and even quasicrystals [18-20]. These predictions are supported by experimental observations on e.g., soft spherical polymers, micelles or dendrons [21-23], as well as polymer-coated nanoparticles [24-26], which all demonstrate a rich crystal phase behavior [27].

In many cases, the complexity of the structures that form in these systems can be understood from the presence of multiple favored length scales: certain particle separations are either favored or disfavored by the shape of the interaction potential. One of the most fundamental examples of such a model is the hard core square shoulder (HCSS) model, in which the spherical particles 
cannot overlap, and pay a fixed energy penalty for approaching each other too closely. In this case, the two length scales are set by the hard-core diameter $\sigma$ and the (larger) interaction range $\sigma+\delta$, as illustrated in Figure 1. Although the HCSS model is not designed to quantitatively model a specific system, it can be seen as a phenomenological model for colloidal particles with a hard core and a soft corona, such as polymer-coated nanoparticles [24,25]. Moreover, it serves as a fundamental model for understanding the phase behavior of models incorporating a competition between two length scales, and, as a result, has received significant attention over the past decades. In two dimensions, such models have been shown to stabilize quasi-crystalline phases [20,28,29], as well as a large variety of crystal lattices [30,31]. In three dimensions, fluid [32] and glass states of the HCSS model have been shown to exhibit unusual behavior, such as polyamorphism and water-like anomalies [33-36]. Additionally, the crystal phase behavior of HCSS models has been studied extensively in the range of shoulder length $\delta / \sigma \in\{0.03, \ldots, 0.08\}$, where an isostructural transition between two face-centered cubic (FCC) structures occurs, ending in a critical point at high temperatures [37-39]. In contrast, for long shoulder lengths $\delta / \sigma>1.5$, particles can self-assemble into highly complex, low-symmetry lattices, forming clusters, columns, or lamellae $[40,41]$. In the intermediate regime, square-shoulder models have been predicted to form a number of crystal structures, including body-centered cubic (BCC) and A15 lattices [17,42], both also observed in experiments of soft repulsive particles [43-45]. However, an exhaustive search of the range of crystal structures that might be stable in this regime at low temperatures is still lacking.

a)

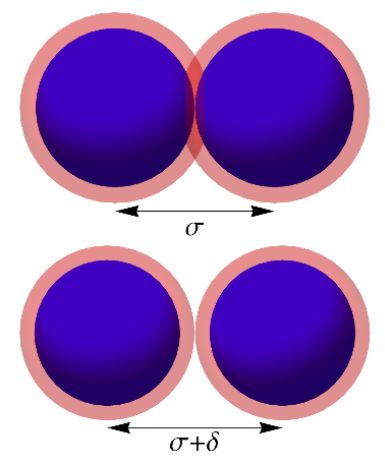

b)

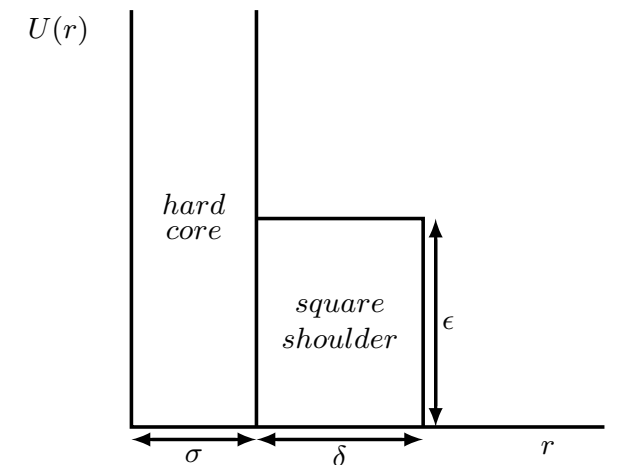

Figure 1. Schematic representation of the hard core square shoulder (HCSS) model. (a) Illustration of two pairs of particles (blue) with their interaction ranges (pink), at distance $\sigma$ (top, energy $U=\epsilon$ ), and at distance $\sigma+\delta$ (bottom, energy $U=0$ ); (b) Plot of the interaction potential.

In this work, we systematically explore the ground-state phase behavior of the three-dimensional HCSS model over a broad range of interaction ranges, and identify a number of stable crystal structures that were not considered in earlier work. Additionally, we draw approximate phase diagrams using a mean-field cell theory for two choices of the interaction range, taking into account the free energies of both fluid and crystalline phases. Our results provide an excellent basis for future studies on e.g., the stability of quasicrystalline phases in three-dimensional HCSS models, as well as the development of more detailed models for e.g., soft-shelled nanoparticles.

The paper is organized as follows. In Section 2, we describe the methods we use to identify candidate crystal structures and to determine the free energy of the fluid and crystal phases. The results are presented in Section 3, where we map out the zero-temperature phase diagram for the HCSS model with shoulder lengths $0<\delta / \sigma<0.41$, and draw the phase diagrams in the density-temperature plane for $\delta / \sigma=0.15$ and 0.2. We present our conclusions and discussion in Section 4. 


\section{Model and Simulation Methods}

The square shoulder potential [37] between a pair of particles can be written as

$$
U(r)= \begin{cases}\infty, & r<\sigma, \\ \epsilon, & \sigma<r<\sigma+\delta, \\ 0, & r>\sigma+\delta,\end{cases}
$$

where $r$ is the distance between the particle centers, $\sigma$ the diameter of the particle and $\epsilon$ the potential of the shoulder with length $\delta$.

In order to study the phase behavior of this model system, we first predict candidate crystal structures using the "floppy-box" Monte Carlo method [46,47], and then determine the free energies of these structures using an approximate mean-field cell theory. Additionally, we use thermodynamic integration to determine the free energy of the fluid phase. From these free energies, we determine the phase diagrams for shoulder widths $\delta / \sigma=0.15$ and 0.2 using common tangent constructions. In the following subsections, we describe the crystal prediction and free energy calculation methods in more detail.

\subsection{Crystal Structure Prediction}

A crucial step in exploring the phase behavior of any colloidal model is the identification of crystal structures that should be taken into account as potentially stable phases. Although, for very simple models, one can often reasonably guess what crystals may be relevant, the prediction of stable structures from an interaction potential is generally far from straightforward. Hence, the search for potentially stable crystal structures is often done by a systematic numerical search, using e.g., genetic algorithms $[31,48,49]$ or simulations of single unit cells [46-48].

Here, we apply the floppy-box Monte Carlo (FBMC) method [46,47]. This method makes use of simulations of extremely small simulation cells with periodic boxes, at constant number of particles $N$, pressure $P$, and temperature $T$. The small number of particles in the box $(N \leq 14)$ allows for rapid sampling of different crystal structures. The three vectors that span the simulation box, vary separately both in length and orientation. During the simulation, we slowly increase the temperature to quench the system into a low-energy state, and examine the resulting structures from a large number of simulations with different choices of $1 \leq N \leq 14$ and $1 \leq P \sigma^{3} / \epsilon \leq 20$. This approach is highly likely to find structures that are stable in the low-temperature limit, where, at any given density, the structure or coexistence of structures with the lowest potential energy is the stable phase. Additionally, structures that are entropically favored are more likely to occur frequently [46]. Hence, we look for structures that are either stable at zero temperature (i.e., have the lowest energy at a given density), or occur repeatedly in our simulations, and use these structures in our free-energy calculations. We identify the symmetry group of the observed crystals using the FINDSYM program (version 5.1.1, ISOTROPY Software Suite, Brigham Young University, Provo, UT, USA) [50].

In addition to the original FBMC approach, we make use of a variation of the same technique that uses shifted boundary conditions, as described in the Supplementary Information. Together with the crystal structures found via these methods, we consider in our calculations the $A 15$ [17] and C14 [24] structures, which have been observed in previous studies of hard-core soft-shell particles.

\subsection{Crystal Free Energies}

To estimate the free energy of the crystal phases, we use an approximate mean-field cell theory [51,52]. In this approach, the free energy of each particle is calculated by assuming that all other particles are located exactly at their lattice sites. The partition function for the particle under consideration can then be calculated numerically by inserting this particle randomly at different 
positions into a sufficiently large volume $V_{0}$ surrounding its lattice position $\mathbf{r}_{0}$, and determining the energy of the particle at that position. Specifically, the partition function $Q_{1}$ for the particle is given by

$$
Q_{1}=\frac{V_{0}}{\Lambda^{3}}\left\langle\exp \left(-\beta\left[u(\mathbf{r})-\frac{1}{2} u\left(\mathbf{r}_{0}\right)\right]\right)\right\rangle_{V_{0}},
$$

where $u(\mathbf{r})$ is the energy of the particle at position $\mathbf{r}, \Lambda$ is the thermal wavelength, and $\beta=1 / k_{B} T$ with $k_{B}$ Boltzmann's constant and $T$ the temperature. Subsequently, the free energy per particle of the crystal is obtained by averaging the single-particle free energy $F_{1}=-k_{B} T \log Q_{1}$ for all particles in the crystal unit cell.

For many crystal structures, the details of the unit cell vary with density and temperature. For example, a body-centered tetragonal (BCT) structure is essentially a body-centered cubic (BCC) crystal compressed in one direction, such that the lattice spacing $c$ along one of the axes is different from the spacing $a$ along the other two. The ratio between the unit cell lengths $c / a$ depends on both the density and temperature, and hence a calculation of the free energy of this structure needs to take this into account. In other crystal structures, the lengths and directions of the vectors controlling the unit cell, and the positions of particles in the cell, may similarly vary. To address this, we need to minimize the crystal free energy with respect to all free parameters at each density and temperature. However, this is computationally expensive when the number of free parameters is large. To speed up this process, we make use of the observation that, in systems of purely hard spheres, the single-particle partition function can be calculated fairly accurately by estimating that the particle can move freely in a polyhedral volume obtained by moving all of the faces of the particle's Voronoi cell inwards by a distance $\sigma / 2$ [17]. Although this approximation slightly underestimates the entropy of the particle, it allows for significantly faster calculations. We extend this approach here to the square shoulder model by approximately dividing the insertion volume up into polyhedral regions with different potential energies, and construct the total single-particle partition function as:

$$
Q_{1}=\frac{1}{\Lambda^{3}} \sum_{i} V_{i} \exp \left(-\beta\left[u_{i}-u\left(\mathbf{r}_{0}\right)\right]\right)
$$

with $u_{i}$ the potential energy in subvolume $i$. We construct the boundaries of all polyhedral subvolumes $V_{i}$ by dividing surfaces obtained by shifting faces of the central particle's Voronoi cell inwards by either $\sigma / 2$ or $(\sigma+\delta) / 2$, and calculate their volumes using the Voro++ library [53]. For more details, see the Supplementary Information. When comparing the Voronoi approach to the insertion approach for identical choices of free parameters, we obtain good agreement, with differences on the order of $0.05 k_{B} T$. The effect of this approximation is of similar magnitude as the errors expected from the mean-field cell theory assumptions.

Note that these free energies are approximate, and hence the predicted phase boundaries are expected to deviate from the true phase diagram, especially at higher temperatures where entropy plays a stronger role. Nonetheless, cell theory has proven to be effective in predicting the phase behavior of systems with both hard and soft interactions $[54,55]$.

\section{Fluid Free Energy}

To determine the free energy of the fluid, we perform Monte Carlo simulations in the NPT ensemble, i.e. at fixed number of particles $N=343$, fixed pressure $P$, and fixed temperature $T$. We perform simulations in a simulation box with fixed cubic shape, for a broad range of pressures and temperatures. We then determine the fluid free energy by using thermodynamic integration [56] of the pressure $P(\rho)$ as a function of the number density $\rho=N / V$ :

$$
\frac{\beta F(\rho)}{N}=\frac{\beta F_{i d}(\rho)}{N}+\int_{0}^{\rho} d \rho^{\prime} \frac{\beta P\left(\rho^{\prime}\right)-\rho^{\prime}}{\rho^{\prime 2}},
$$


where $F_{i d}(\rho)=N k_{b} T\left(\log \rho \Lambda^{3}-1\right)$ is the ideal gas free energy. When fitting the equation of state, we improve accuracy at low densities by making use of the second virial coefficient $B_{2}$, which can be calculated analytically and is given by

$$
B_{2}=-\frac{2 \pi}{3}(\sigma+\delta)^{3}\left(e^{-\beta \epsilon}-1\right)+\frac{2 \pi}{3} \sigma^{3} e^{-\beta \epsilon} .
$$

\section{Results}

We begin our investigation by exploring the phase behavior of the square shoulder model in the zero-temperature limit. To this end, we use FBMC simulations to obtain candidate crystal structures for a range of choices of the interaction range $\delta$, and collect the dimensionless number density $\rho \sigma^{3}$ and potential energy per unit volume $E=\beta U \sigma^{3} / V$ of each structure for fixed $\delta$ in a diagram such as the one shown in Figure 2. In this representation, the potential energy of a coexisting state between two crystals is represented as a straight tie-line between the points corresponding to these crystals. Since at each density, the most stable state in the zero-temperature limit is the phase or coexistence of two phases with the lowest potential energy, the zero-temperature phase diagram can be obtained by simply connecting the lowest points at each density (effectively a common tangent construction), as shown in Figure 2 for the interaction range $\delta / \sigma=0.2$.

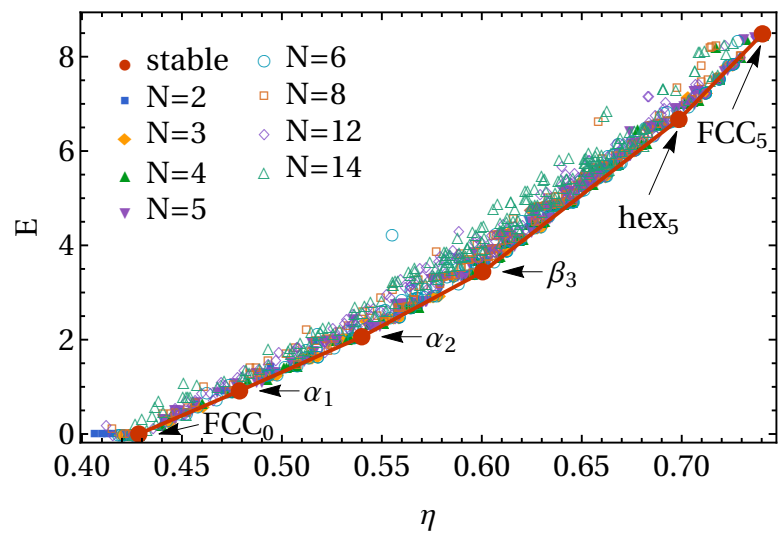

Figure 2. Candidate crystal structures for interaction range $\delta / \sigma=0.2$. Each point represents the density $\rho$ and potential energy per unit volume $E=\beta U \sigma^{3} / V$ of the final structure in an independent floppy-box Monte Carlo simulation. Simulations were run with varying choices for the number of particles $N$, temperature, and pressure. Note that, for all stable structures, the same crystal was found from multiple independent simulations.

For this interaction range, we find six distinct stable crystal structures at zero temperature, differentiated by their potential energy per particle and density. With increasing density, we first encounter $\mathrm{FCC}_{0}$, a low-density face-centered cubic phase (see Figure 3), with the subscript indicating the potential energy per particle $u=U / N \epsilon=0$. This crystal consists of low-density hexagonal planes (lattice spacing $\sigma+\delta$ ), where particles interact neither with neighbors in the same plane, nor with neighbors in adjacent planes. Next, we obtain a crystal structure consisting of distorted hexagonal planes that we call $\alpha$-planes, in which each particle interacts with two neighbors within the plane. These planes are stacked such that particles in neighboring planes do not interact (while maximizing the density), leading to a total energy per particle $u=1$. The resulting crystal structure, which we call $\alpha_{1}$, is commensurate with a body-centered orthogonal (BCO) lattice. The next structure, $\alpha_{2}$, consists of the same planes, but has each particle interacting with an additional neighbor in each adjacent plane, leading body-centered tetragonal (BCT) lattice with a total of four bonds per particle $(u=2)$. Moving on to higher densities, we find a crystal structure consisting of $\beta$-planes: hexagonal planes distorted such that particles now interact with four in-plane neighbors. It should be noted that, when these planes are stacked such that they form no out-of-plane bonds, we simply recover the $\alpha_{2}$ crystal 
in a different orientation. In the $\beta_{3}$ structures, the $\beta$-planes are instead stacked such that each particle interacts with one particle in each neighboring plane. Finally, in the high-density regime, we again find hexagonal planes, but this time with a lattice spacing of $\sigma$, such that each particle interacts with six neighbors within the same plane. These planes can again be stacked to allow for a different number of out-of-plane bonds, resulting in a body-centered orthogonal lattice hex ${ }_{5}$ with a total energy of $u=5$, and, finally hex 6 , corresponding to densely packed FCC, with total energy $u=6$.

\begin{tabular}{|c|c|c|c|c|}
\hline Name & Front view & Side view & $U / N \epsilon$ & Notes \\
\hline $\mathrm{FCC}_{0}$ & & & 0 & Low-density FCC. \\
\hline$\alpha_{1}$ & & & 1 & $\begin{array}{l}\text { Body-centered orthogonal } \\
\text { (BCO) lattice. Also contains } \\
\text { low-density hexagonal planes. }\end{array}$ \\
\hline$\alpha_{2}$ & & & 2 & $\begin{array}{l}\text { Body-centered tetragonal } \\
\text { lattice. Also contains square } \\
\text { and } \beta \text { planes. }\end{array}$ \\
\hline$\beta_{3}$ & & & 3 & Also contains $\alpha$ planes. \\
\hline$\beta_{4}$ & & & 4 & $\begin{array}{l}\text { Body-centered tetragonal } \\
\text { (BCT) lattice. Body-centered } \\
\text { cubic when } \delta=\frac{2}{\sqrt{3}}-1 \simeq 0.1547 \text {. }\end{array}$ \\
\hline $\operatorname{hex}_{4}$ & & & 4 & Also contains $\beta$ planes. \\
\hline $\operatorname{hex}_{5}$ & & & 5 & $\begin{array}{l}\text { Body-centered orthogonal } \\
\text { (BCO) lattice. }\end{array}$ \\
\hline $\mathrm{FCC}_{6}$ & & & 6 & High-density FCC. \\
\hline
\end{tabular}

Figure 3. Graphical representations of stable ground-state crystal structures. For each crystal, we list a name, two snapshots with different planes highlighted in different colors, and any remarks on the details of the structure. In all snapshots, $\delta / \sigma=0.2$, with the exception of $\beta_{4}$ (with $\delta / \sigma=0.15$ ) and hex $_{4}$ (with $\delta / \sigma=0.25$ ).

It is important to note that these structures can all be modified by altering the stacking of consecutive layers. For example, while the simplest way of stacking hexagonal planes leads to an FCC structure, an alternate stacking choice leads to a hexagonally close-packed (HCP) crystal phase. In principle, an infinite number of distinct stackings is possible, all with the same potential energy and density. Similarly, for the other phases identified here, there are always multiple choices for placing consecutive layers, which correspond to different crystal structures. Since these are all equally stable at zero temperature, and our cell theory approach would not correctly capture free energy differences between them at finite temperatures, in this work, we always assume the simplest possible stacking (corresponding to the smallest unit cell) in each case. Similarly, crystal structures containing identical planes can in principle be mixed by varying the way these planes are stacked. This essentially 
corresponds to a coexistence between the two phases. At zero temperature, there is no surface tension cost for these mixed stackings, and as such they are equally stable as a coexistence of the pure phases. At finite temperatures, however, we expect these mixed stackings to carry a finite interfacial free energy cost, and hence we only consider the pure phases for our phase diagrams.

We repeat our crystal search procedure for a broad range of interaction ranges $0<\delta / \sigma=0<0.41$. Note that $\delta / \sigma=\sqrt{2}-1 \simeq 0.41$ marks the point where diagonal neighbors in a close-packed square arrangement start interacting. As a result, a number of the crystal structures predicted here are expected to change when $\delta$ is increased beyond this point. In the investigated regime, we found two

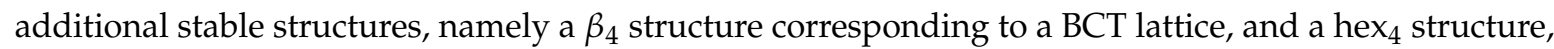
both with an energy $u=4$.

In Figure 3, we list all obtained structures and provide snapshots from two different angles. Although we classify the structures by breaking them down into approximately hexagonal planes, several of them have additional symmetries which allow for a more precise identification of the structure. In particular, as listed in Figure 3 , the $\alpha_{1}$ and hex ${ }_{5}$ crystals correspond to a body-centered orthogonal (BCO) lattice, and the $\alpha_{2}$ and $\beta_{4}$ crystals correspond to a BCT lattice. Interestingly, BCO lattices have been previously predicted for soft repulsive particles, such as star polymers $[15,57,58]$.

Combining our information on all stable structures, we systematically map out the phase diagram in the zero-temperature limit, for interaction ranges $0<\delta / \sigma<0.4$, and plot the result in Figure 4 . We also include here the stability regime of the fluid phase. At low densities, where packings with zero energy are possible, the system simply acts as a system of hard spheres of diameter $\sigma+\delta$, exhibiting a fluid-FCC coexistence between densities $0.94<\rho(\sigma+\delta)^{3}<1.04$ [59]. Other crystal structures only show up beyond the maximum density for the low-density FCC phase $\left(\rho(\sigma+\delta)^{3}=\sqrt{2}\right)$. Note that, at zero temperature, these higher-energy structures only appear at their maximum density, in order to allow as much of the system to remain in the lower-energy state as possible. This results in a phase diagram mostly filled by (white) coexistence regions, where the system is expected to macroscopically phase separate into the two adjacent phases. For example, at $\delta=0.2$ and $\rho \sigma^{3}=0.57$ (located in the fluid-FCC coexistence region in Figure 4), we expect the system to be divided into a fluid region at the freezing density of $\rho_{f} \sigma^{3}=0.54$ and a low-density FCC region at the melting density of $\rho_{x} \sigma^{3}=0.60$.

Even in the limit of zero temperature, the HCSS model shows a surprisingly rich phase diagram, with up to seven different phases stable for a given $\delta$. Interestingly, many of these structures have not been considered in earlier studies exploring the phase behavior of square-shoulder models. All stable structures identified here can be represented by a unit cell containing only a single particle. Note that, apart from stacking variations, the FBMC simulations yielded no high frequencies of any structures beyond those stable at zero temperature, and the C14 and $A 15$ lattices were not found to be stable in the zero-temperature limit.

While Figure 4 shows the structures which could, in principle, be found in the limit of strong interactions, the influence of entropy at finite temperatures can be expected to significantly change the phase behavior. To explore the effects of finite temperature, we focus on two values of the interaction range, $\delta / \sigma=0.15$ and 0.2 , and map out the phase behavior as a function of temperature and density using cell theory. Here, we consider all crystal structures found in the zero-temperature limit, as well as the $C 14$ and $A 15$ lattices.

In Figure 5a, we show the phase diagram in the density-temperature plane for interaction range $\delta=0.15$, as obtained from the free energies calculated using cell theory. Along the bottom axis, we recover the zero-temperature limit, showing a succession of phases including the fluid, low-density $\mathrm{FCC}_{0}, \alpha_{1}, \alpha_{2}, \beta_{4}$, and high-density $\mathrm{FCC}_{6}$ phases. Note that the $\beta_{4}$ rapidly and continuously transforms into a BCC crystal at finite temperatures, and is therefore labeled as BCC in the phase diagram. Although the $\alpha_{1}$ and $\alpha_{2}$ structures show only small regions of stability, both FCC phases and the BCC phase are stable in significant parts of the phase diagram. Interestingly, the fluid phase shows reentrant behavior close to the temperature where the low-density FCC phase vanishes. This results in a small regime where the FCC phase can melt upon both compression and expansion. With increasing 
temperature, the density range in which this occurs narrows and eventually vanishes at a single point, beyond which the low-density FCC phase is no longer stable. At sufficiently high temperatures, we recover the simple fluid-FCC coexistence expected in the high-temperature limit, which corresponds to the pure hard-sphere model.

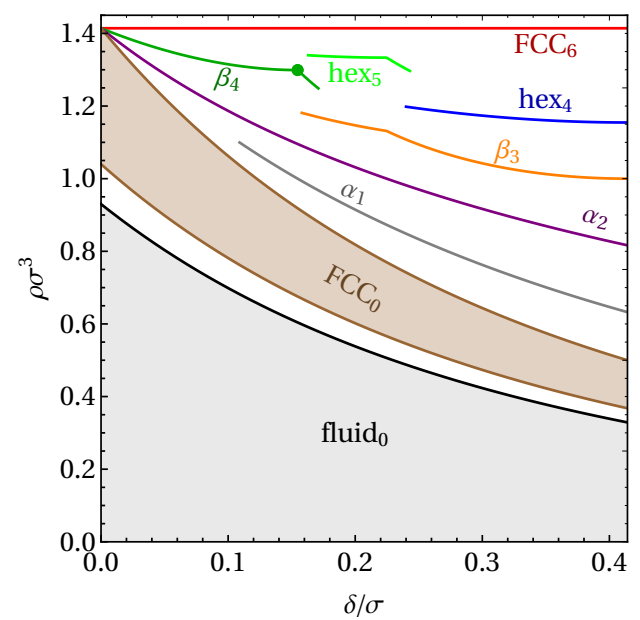

Figure 4. Phase diagram of the hard core square shoulder model in the zero-temperature limit, as a function of interaction range $\delta$ and number density $\rho$. The white areas indicate coexistence regions. All tie lines between coexisting state points are vertical. The green point marks the value of $\delta / \sigma$ where the ground-state $\beta_{4}$ corresponds to a body-centered cubic (BCC) lattice.

We show the phase diagram for interaction range $\delta / \sigma=0.2$ in Figure $5 \mathrm{~b}$. We observe similar phase behavior, where again only the phases stable at zero temperature occur in the phase diagram. In contrast to the findings in Ref. [17], we find no stable BCC or A15 phase, as both phases are metastable with respect to melting. This discrepancy can likely be attributed to the fact that, in Ref. [17], the stability of the fluid phase at non-zero temperatures was estimated by assuming that the freezing density remained the same as in the zero-temperature limit. Our results thus highlight the importance of considering the enhanced stability of the fluid at non-zero temperatures. However, we cannot exclude the possibility of a stable $A 15$ phase at larger $\delta$, where Ref. [17] predicts a larger region of stability for this structure.
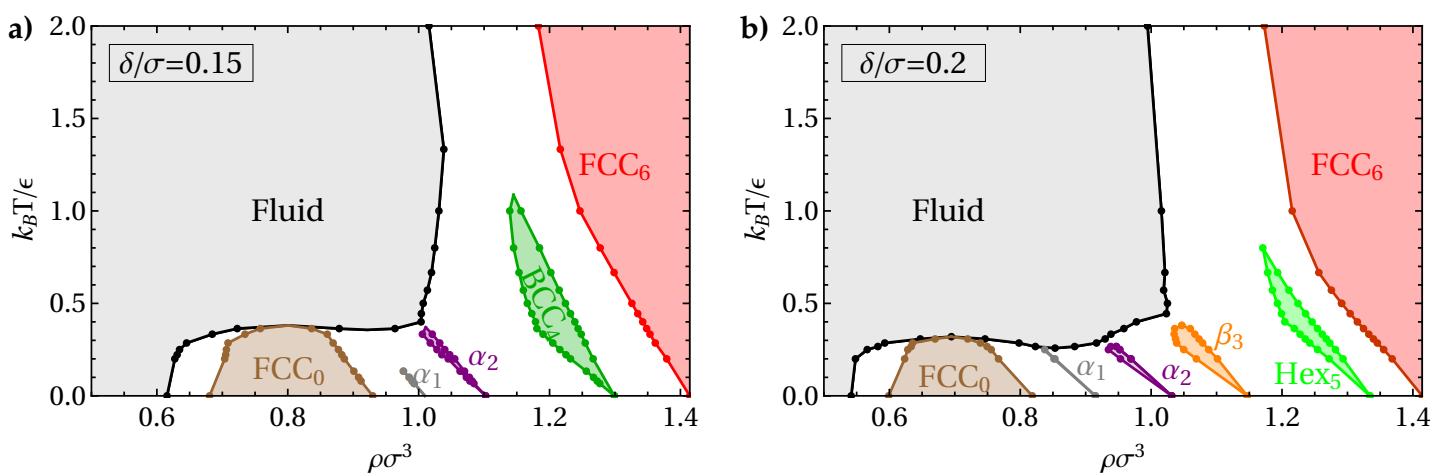

Figure 5. Phase diagrams for interaction ranges $\delta / \sigma=0.15$ (a) and $0.2(\mathbf{b})$, as a function of density $\rho$ and temperature $T$. The white areas indicate coexistence regions. All tie lines between coexisting state points are horizontal. Coexistences (points) are obtained from free energies calculated using cell theory. Lines are guides to the eye only. Triple points are obtained by extrapolating coexistence lines.

\section{Conclusions}

We systematically explored the low-temperature phase behavior of the hard-core square-shoulder model, identifying a rich variety of distinct crystal structures that are stable in the zero-temperature 
limit. Additionally, we constructed phase diagrams in the density-temperature plane for interaction ranges $\delta / \sigma=0.15$ and 0.2 using cell theory, in order to estimate the temperature regimes in which these structures may be observed. Although all crystal structures identified here are relatively simple, consisting of unit cells containing only a single particle, their importance cannot be intuitively predicted a priori, illustrating the importance of a systematic search for unexpected stable structures.

Our results highlight the unexpectedly complex phase behavior that can result from the fundamental HCSS model. Moreover, it demonstrates that a small change in interaction range from $\delta / \sigma=0.15$ to 0.2 can both stabilize and destabilize several crystal structures. This sensitivity also implies that small variations in the interaction potential shape, such as smoothening the sharp potential jump associated with the shoulder, can be expected to have far-reaching effects. Hence, it is not surprising that more realistic models for hard-core soft-shell particles have predicted a variety of crystal structures not seen here, including e.g., $C 14$ and $A 15$ phases [60]. Such phases are typically explained in terms of a minimization of the surface energy between building blocks [17], resulting from e.g., the elasticity of the particle surface layers. In contrast, in the HCSS model, phases are stabilized purely due to the geometric interplay between the hard-core and soft-shoulder length scales, which leads to the stabilization of a distinct set of crystal structures. While this model potential is not expected to be quantitatively accurate for any specific colloidal system, it provides a framework for understanding the crystallization of colloids that interact differently at two length scales. However, quantitative predictions for the phase behavior of real colloidal systems will require carefully tuned model potentials, tailored to the details of the experimental system under consideration.

Although all predicted structures have small unit cells, it should be noted that our approach cannot exclude the possibility of stable crystals with larger unit cells than those considered in our FBMC simulations, or the possibility of stable quasicrystals, which are not commensurate with a periodic unit cell. In particular, quasicrystalline layers have recently been shown to form in a sedimenting HCSS system with shoulder length $\delta / \sigma=0.4$ [61]. By providing a clear overview of competing periodic structures, the phase behavior predicted here provides important guidelines for exploring the stability of quasicrystalline or other non-periodic structures in this fundamental model.

As an outlook, we emphasize that the rich equilibrium phase diagram obtained here for the square-shoulder model provides the starting point for future studies of guided colloidal self-assembly. The variety of stable crystal structures presented in this work may constitute important building blocks for photonic $[62,63]$ and phononic $[64,65]$ crystals with unusual material properties. Several techniques are conceivable to produce these exotic structures, including colloidal templating $[66,67]$ and field-directed crystallization [68]. In the first case, a stable but kinetically blocked crystalline structure is forced to occur by an external template that incorporates the symmetry of the final desired equilibirum crystal structure. In the second case, a time-dependent external field produces dynamical channels to force the system to relax into the desired crystal. For both approaches, a detailed understanding of the equilibrium phase behavior, and the presence of competing crystal structures, is crucial. Hence, the phase diagrams and crystal structures predicted here may pave the way towards the design of new colloidal crystal structures for various applications.

Supplementary Materials: The following are available online at www.mdpi.com/1996-1944/10/11/1280/s1, supplementary information: SI.pdf.

Acknowledgments: We gratefully acknowledge support from the Deutsche Forschungsgemeinschaft (Grant LO 418/19-1).

Author Contributions: F.S. conceived and designed the simulations; A.G. performed the simulations; A.G. and F.S. analyzed the data; A.G., H.L., and F.S. wrote the paper.

Conflicts of Interest: The authors declare no conflict of interest. 


\section{References}

1. Frenkel, D.; Wales, D.J. Colloidal self-assembly: Designed to yield. Nat. Mater. 2011, 10, 410-411.

2. Vogel, N.; Retsch, M.; Fustin, C.A.; del Campo, A.; Jonas, U. Advances in colloidal assembly: The design of structure and hierarchy in two and three dimensions. Chem. Rev. 2015, 115, 6265-6311.

3. Zhang, Y.; Lu, F.; Yager, K.G.; Van Der Lelie, D.; Gang, O. A general strategy for the DNA-mediated self-assembly of functional nanoparticles into heterogeneous systems. Nat. Nanotechnol. 2013, 8, 865-872.

4. Kodger, T.E.; Guerra, R.E.; Sprakel, J. Precise colloids with tunable interactions for confocal microscopy. Sci. Rep. 2015, 5, doi:10.1038/srep14635.

5. Yethiraj, A.; van Blaaderen, A. A colloidal model system with an interaction tunable from hard sphere to soft and dipolar. Nature 2003, 421, 513-517.

6. Löwen, H. Introduction to colloidal dispersions in external fields. Eur. Phys. J. Spec. Top. 2013, 222, $2727-2737$.

7. Grzelczak, M.; Vermant, J.; Furst, E.M.; Liz-Marzán, L.M. Directed self-assembly of nanoparticles. ACS Nano 2010, 4, 3591-3605.

8. Dinsmore, A.D.; Crocker, J.C.; Yodh, A.G. Self-assembly of colloidal crystals. Curr. Opin. Colloid Interface Sci. 1998, 3, 5-11.

9. Palberg, T. Crystallization kinetics of repulsive colloidal spheres. J. Phys. Condens. Matter 1999, 11, R323.

10. Ivlev, A.; Löwen, H.; Morfill, G.; Royall, C.P. Complex Plasmas and Colloidal Dispersions: Particle-Resolved Studies of Classical Liquids and Solids; World Scientific, Series in Soft Condensed Matter; World Scientific Publishing: Hackensack, NJ, USA, 2012; Volume 5.

11. Meijer, J.M.; Hagemans, F.; Rossi, L.; Byelov, D.V.; Castillo, S.I.; Snigirev, A.; Snigireva, I.; Philipse, A.P.; Petukhov, A.V. Self-assembly of colloidal cubes via vertical deposition. Langmuir 2012, 28, 7631-7638.

12. Vroege, G.J.; Lekkerkerker, H.N. Phase transitions in lyotropic colloidal and polymer liquid crystals. Rep. Prog. Phys. 1992, 55, 1241.

13. Op Reinink, A.L.; van den Pol, E.; Petukhov, A.; Vroege, G.; Lekkerkerker, H. Phase behaviour of lyotropic liquid crystals in external fields and confinement. Eur. Phys. J. Spec. Top. 2013, 222, 3053-3069.

14. Lahtinen, T.; Haataja, J.S.; Tero, T.R.; Häkkinen, H.; Ikkala, O. Template-Free Supracolloidal Self-Assembly of Atomically Precise Gold Nanoclusters: From 2D Colloidal Crystals to Spherical Capsids. Angew. Chem. Int. Ed. 2016, 55, 16035-16038.

15. Watzlawek, M.; Likos, C.N.; Löwen, H. Phase diagram of star polymer solutions. Phys. Rev. Lett. 1999, 82,5289 .

16. Likos, C.N.; Hoffmann, N.; Löwen, H.; Louis, A.A. Exotic fluids and crystals of soft polymeric colloids. J. Phys. Condens. Matter 2002, 14, 7681.

17. Ziherl, P.; Kamien, R.D. Maximizing Entropy by Minimizing Area: Towards a New Principle of Self-Organization. J. Phys. Chem. B 2001, 105, 10147-10158.

18. Barkan, K.; Diamant, H.; Lifshitz, R. Stability of quasicrystals composed of soft isotropic particles. Phys. Rev. B 2011, 83, 172201.

19. Denton, A.; Löwen, H. Stability of colloidal quasicrystals. Phys. Rev. Lett. 1998, 81, 469.

20. Dotera, T.; Oshiro, T.; Ziherl, P. Mosaic two-lengthscale quasicrystals. Nature 2014, 506, $208-211$.

21. Sakya, P.; Seddon, J.M.; Templer, R.H.; Mirkin, R.J.; Tiddy, G.J.T. Micellar cubic phases and their structural relationships: The nonionic surfactant system C12EO12/water. Langmuir 1997, 13, 3706-3714.

22. Ungar, G.; Liu, Y.; Zeng, X.; Percec, V.; Cho, W.D. Giant supramolecular liquid crystal lattice. Science 2003, 299, 1208-1211.

23. Lee, S.; Leighton, C.; Bates, F.S. Sphericity and symmetry breaking in the formation of Frank-Kasper phases from one component materials. Proc. Natl. Acad. Sci. USA 2014, 111, 17723-17731.

24. Hajiw, S.; Pansu, B.; Sadoc, J.F. Evidence for a C14 Frank-Kasper phase in one-size gold nanoparticle superlattices. ACS Nano 2015, 9, 8116-8121.

25. Goodfellow, B.W.; Rasch, M.R.; Hessel, C.M.; Patel, R.N.; Smilgies, D.M.; Korgel, B.A. Ordered structure rearrangements in heated gold nanocrystal superlattices. Nano Lett. 2013, 13, 5710-5714.

26. Kuttner, C.; Chanana, M.; Karg, M.; Fery, A. Macromolecular Decoration of Nanoparticles for Guiding Self-Assembly in 2D and 3D; Wiley-VCH: Weinheim, Germany, 2016.

27. Boles, M.A.; Engel, M.; Talapin, D.V. Self-assembly of colloidal nanocrystals: From intricate structures to functional materials. Chem. Rev. 2016, 116, 11220-11289. 
28. Pattabhiraman, H.; Gantapara, A.P.; Dijkstra, M. On the stability of a quasicrystal and its crystalline approximant in a system of hard disks with a soft corona. J. Chem. Phys. 2015, 143, 164905.

29. Pattabhiraman, H.; Dijkstra, M. Phase behaviour of quasicrystal forming systems of core-corona particles. J. Chem. Phys. 2017, 146, 114901.

30. Malescio, G.; Pellicane, G. Stripe phases from isotropic repulsive interactions. Nat. Mater. 2003, 2, 97-100.

31. Fornleitner, J.; Kahl, G. Pattern formation in two-dimensional square-shoulder systems. J. Phys. Condens. Matter 2010, 22, 104118.

32. Yuste, S.B.; Santos, A.; López de Haro, M. Structure of the square-shoulder fluid. Mol. Phys. 2011, 109, 987-995.

33. Buldyrev, S.V.; Malescio, G.; Angell, C.A.; Giovambattista, N.; Prestipino, S.; Saija, F.; Stanley, H.E.; $\mathrm{Xu}, \mathrm{L}$. Unusual phase behavior of one-component systems with two-scale isotropic interactions. J. Phys. Condens. Matter 2009, 21, 504106.

34. Heyes, D.M.; Aston, P.J. Square-well and square-shoulder fluids: Simulation and equations of state. J. Chem. Phys. 1992, 97, 5738-5748.

35. Bordin, J.R.; Barbosa, M.C. Brownian dynamics leads to two waterlike anomalous regions in a 2D core-softened potential. arXiv 2017, arXiv:1709.05512.

36. Sperl, M.; Zaccarelli, E.; Sciortino, F.; Kumar, P.; Stanley, H.E. Disconnected Glass-Glass Transitions and Diffusion Anomalies in a model with two repulsive length scales. Phys. Rev. Lett. 2010, 104, 145701.

37. Kincaid, J.M.; Stell, G.; Goldmark, E. Isostructural phase transitions due to core collapse. II. A three-dimensional model with a solid-solid critical point. J. Chem. Phys. 1976, 65, 2172-2179.

38. Bolhuis, P.; Frenkel, D. Isostructural solid-solid transitions in systems with a Repulsiveshoulder'potential. J. Phys. Condens. Matter 1997, 9, 381-387.

39. Denton, A.; Löwen, H. Isostructural solid-solid transitions in square-shoulder systems. J. Phys. Condens. Matter 1997, 9, L1.

40. Pauschenwein, G.J.; Kahl, G. Clusters, columns, and lamellae-Minimum energy configurations in core softened potentials. Soft Matter 2008, 4, 1396-1399.

41. Pauschenwein, G.J.; Kahl, G. Zero temperature phase diagram of the square-shoulder system. J. Chem. Phys. 2008, 129, 174107.

42. Rascón, C.; Velasco, E.; Mederos, L.; Navascués, G. Phase diagrams of systems of particles interacting via repulsive potentials. J. Chem. Phys. 1997, 106, 6689-6697.

43. Schmitt, J.; Hajiw, S.; Lecchi, A.; Degrouard, J.; Salonen, A.; Impéror-Clerc, M.; Pansu, B. Formation of Superlattices of Gold Nanoparticles Using Ostwald Ripening in Emulsions: Transition from fcc to bcc Structure. J. Phys. Chem. B 2016, 120, 5759-5766.

44. Li, Y.; Lin, S.T.; Goddard, W.A. Efficiency of various lattices from hard ball to soft ball: Theoretical study of thermodynamic properties of dendrimer liquid crystal from atomistic simulation. J. Am. Chem. Soc. 2004, $126,1872-1885$.

45. Imai, M.; Yoshida, I.; Iwaki, T.; Nakaya, K. Static and dynamic structures of spherical nonionic surfactant micelles during the disorder-order transition. J. Chem. Phys. 2005, 122, 044906.

46. Filion, L.; Marechal, M.; van Oorschot, B.; Pelt, D.; Smallenburg, F.; Dijkstra, M. Efficient method for predicting crystal structures at finite temperature: variable box shape simulations. Phys. Rev. Lett. 2009, 103, 188302.

47. De Graaf, J.; Filion, L.; Marechal, M.; van Roij, R.; Dijkstra, M. Crystal-structure prediction via the Floppy-Box Monte Carlo algorithm: Method and application to hard (non) convex particles. J. Chem. Phys. 2012, 137, 214101.

48. Bianchi, E.; Doppelbauer, G.; Filion, L.; Dijkstra, M.; Kahl, G. Predicting patchy particle crystals: Variable box shape simulations and evolutionary algorithms. J. Chem. Phys. 2012, 136, 214102.

49. Gottwald, D.; Kahl, G.; Likos, C.N. Predicting equilibrium structures in freezing processes. J. Chem. Phys. 2005, 122, 204503.

50. Stokes, H.T.; Hatch, D.M. FINDSYM: Program for identifying the space-group symmetry of a crystal. J. Appl. Crystallogr. 2005, 38, 237-238.

51. Prestipino, S.; Saija, F.; Malescio, G. The zero-temperature phase diagram of soft-repulsive particle fluids. Soft Matter 2009, 5, 2795-2803.

52. Lennard-Jones, J.; Devonshire, A. Critical phenomena in gases. I. Proc. R. Soc. A 1937, 163, 53-70. 
53. Rycroft, C.H. Voro ${ }^{++}$: A three-dimensional Voronoi cell library in $\mathrm{C}^{+}$. Chaos 2009, 19, 041111.

54. Vega, C.; Monson, P.A. Solid-liquid equilibrium for quadrupolar molecules. Mol. Phys. 1995, 85, 413-421.

55. Cottin, X.; Monson, P.A. A cell theory for solid solutions: Application to hard sphere mixtures. J. Chem. Phys. 1993, 99, 8914-8921.

56. Frenkel, D.; Smit, B.; Tobochnik, J.; McKay, S.R.; Christian, W. Understanding molecular simulation. Comput. Phys. 1997, 11, 351-354.

57. Likos, C.N.; Hoffmann, N.; Jusufi, A.; Löwen, H. Interactions and phase behaviour of polyelectrolyte star solutions. J. Phys. Condens. Matter 2002, 15, S233.

58. Hoffmann, N.; Likos, C.N.; Löwen, H. Structure and phase behavior of polyelectrolyte star solutions. J. Chem. Phys. 2004, 121, 7009-7021.

59. Noya, E.G.; Vega, C.; de Miguel, E. Determination of the melting point of hard spheres from direct coexistence simulation methods. J. Chem. Phys. 2008, 128, 154507.

60. Pansu, B.; Sadoc, J.F. Metallurgy of soft spheres with hard core: From BCC to Frank-Kasper phases. arXiv 2017, arXiv:1707.07897 .

61. Pattabhiraman, H.; Dijkstra, M. Periodic layers of a dodecagonal quasicrystal and a floating hexagonal crystal in sedimentation-diffusion equilibria of colloids. J. Chem. Phys. 2017, 147, 104902.

62. Cai, Z.; Liu, Y.J.; Lu, X.; Teng, J. Fabrication of well-ordered binary colloidal crystals with extended size ratios for broadband reflectance. ACS Appl. Mater. Interfaces 2014, 6, 10265-10273.

63. Cai, Z.; Xiong, Z.; Lu, X.; Teng, J. In situ gold-loaded titania photonic crystals with enhanced photocatalytic activity. J. Mater. Chem. A 2014, 2, 545-553.

64. Cheng, W.; Wang, J.; Jonas, U.; Fytas, G.; Stefanou, N. Observation and tuning of hypersonic bandgaps in colloidal crystals. Nat. Mater. 2006, 5, 830-836.

65. Baumgartl, J.; Zvyagolskaya, M.; Bechinger, C. Tailoring of phononic band structures in colloidal crystals. Phys. Rev. Lett. 2007, 99, 205503.

66. Van Blaaderen, A.; Ruel, R.; Wiltzius, P. Template-directed colloidal crystallization. Nature 1997, 385, 321.

67. Velikov, K.P.; Christova, C.G.; Dullens, R.P.A.; van Blaaderen, A. Layer-by-layer growth of binary colloidal crystals. Science 2002, 296, 106-109.

68. Bauer, J.L.; Kurian, M.J.; Stauffer, J.; Furst, E.M. Suppressing the Rayleigh-Plateau Instability in Field-Directed Colloidal Assembly. Langmuir 2016, 32, 6618-6623.

(C) 2017 by the authors. Licensee MDPI, Basel, Switzerland. This article is an open access article distributed under the terms and conditions of the Creative Commons Attribution (CC BY) license (http:/ / creativecommons.org/licenses/by/4.0/). 